allosteric. equilibrium of high-spin methaemoglobin derivatives (aquo-, fluoro-) in favour of the $T$ (deoxy) state. The reduced reactivity of the $\beta$-93 sulphydryl groups is also comparable with that observed for deoxy as compared with oxy-haemoglobin. IHP seems to bind to the same site in met- as in deoxy-haemoglobin, between the terminal amino groups of the $\beta$ chains. Smaller changes in the UV spectra and $\mathrm{SH}$ group reactivities are induced in low-spin methaemoglobin derivatives (azido-, cyano-) by IHP without the sign reversal in the $\mathrm{CD}$ spectrum at $287 \mathrm{~nm}$ associated with the $R \rightarrow T$ transition of deoxyhaemoglobin. Similarly, the NMR studies show that for the high-spin derivatives IHP causes larger changes in the ring-current-shifted resonances of some aromatic residues than it does for the low-spin derivatives. Taken as a whole, these observations indicate that solutions of high-spin methaemoglobin derivatives contain both $\mathbf{R}$ and $T$ forms in equilibrium, with the $R$ form favoured by high $p \mathrm{H}$, and the $\mathrm{T}$ form by low $p \mathrm{H}$ and the binding of organic phosphates. This scheme can account qualitatively for the $p H$ dependence of the redox equilibrium of haemoglobin and its sensitivity to chemical modification, and there is an interesting interpretation of the redox data in allosteric terms.

Part 3 , by Perutz, Heidner, Ladner, Beetlestone and Slade, considers changes in the visible and near-IR absorption spectra, paramagnetic susceptibility, paramagnetically - shifted proton-NMR and electron spin resonances induced in high- and low-spin methaemoglobin derivatives by IHP, and relates them ultimately to changes in the distances between the iron atom and its ligands. The increased $\mathrm{Fe}-\mathrm{N}$ bond distances observed in the $\mathrm{T}$ structure implies that the globin exercises tension on the haem which pulls the iron atoms further from the porphyrin ring plane, opposing the transition to the low-spin state needed for combination with oxygen and hence reducing the oxygen affinity. Finally, since haem-haem interaction is observed only when a change in quaternary structure accompanies reaction with ligands, the present findings imply that it is coupled to a change in tension at the haem, transmitted by a change in quaternary structure of the globin. This brings the discussion back to globin, which is the starting point of the series. It should perhaps be added that comprehension of the large amount of diverse experimental data is greatly helped by some summary tables and figures en route which summarise particular conclusions and implications reached during the course of the tightly argued discussion.

\section{Mitotic spindle assembly in vitro}

\section{from a Correspondent}

Two main approaches have been taken in studies of the mitotic spindle. Measurements of the birefringence of the spindle in intact cells by Inoue and others have suggested that the fibrous components of the spindle are in dynamic equilibrium with subunits. The birefringence of the spindle is lost when living cells are exposed to cold, high hydroastatic pressures and to colchicine and other antimitotic alkaloids. These agents are thought to depolymerise reversibly the microtubules of the spindle fibres and so prevent chromosome movement in intact cells. The other approach has been to isolate the intact mitotic apparatus, usually in the presence of stabilising agents such as glycols. This procedure has provided useful information about the chemistry of the mitotic apparatus but not how it works, because reversible assembly and disassembly have not been achieved with spindles so prepared.

Three groups of investigators have now reported the preparation of mitotic spindles which can be made to increase or decrease in size by appropriate treatment of the subcellular materials. The spindles are isolated in the presence of added rat brain tubulin and agents which chelate calcium, thereby preventing the disassembly of spindle microtubules. Cande, Snyder, Smith, Summers and McIntosh (Proc. natn. Acad. Sci. U.S.A., 71, 1559-1563; 1974) lysed rat kangaroo celis with the detergent Triton X-100 into a solution of polymerisable rat brain tubulin and obtained spindles which lose and gain birefringence when cooled and warmed; the spindles can move anaphase chromosomes to the opposite ends of cell preparations. Early anaphase cells lysed into buffers containing polyethylene glycol and nucleotide triphosphates showed spindle elongation and chomosome movement in the absence of added tubulin subunits. The authors conclude that although spindle growth requires microtubule polymerisation, anaphase motions do not.

Rebhun, Rosenbaum, Lefebvre and Smith (Nature, 249, 113-115; 1974) report that spindles isolated from eggs of the surf clam (Spisula) cooled and then warmed with chick brain tubulin showed increased birefringence in the form of fibres similar in distribution to the mitotic apparatus of living cells. Sometimes such artificially polymerised spindles were larger than normal spindles. The isolated spindles increased in length but did not shorten and were stable to dilution of tubulin. It seems that they can assemble tubulin more readily than they can break down the assembled units.

Inoue, Borisy and Kiehart (J. Cell Biol., 62, 175-184; 1974) found that tubulin purified from porcine brain augmented the birefringence of Chaetopterus spindles. The cells were lysed in a hypotonic calcium-chelating solution, and in the presence of tubulin, spindles and asters grew considerably larger than those in intact cells. The isolated augmented spindles depolymerised rapidly at $6^{\circ} \mathrm{C}$, and birefringence was slowly recovered upon return to $23^{\circ} \mathrm{C}$. The birefringence of the spindles also decreased in the presence of $2.5 \mathrm{mM}$ calcium but not on dilution of tubulin or in the presence of colchicine or colcemid.

The results represent a useful advance in knowledge of the mitotic apparatus. They show that the conditions required for polymerisation of isolated spindle microtubules are similar to those defined by Weisenberg and others for tubulin from various sources, including surf clam eggs. The response of the isolated spindles to temperature reflect those observed in intact cells, but other responses, such as those to colchicine do not. Disassembly does not follow dilution of tubulins, so that a simple equilibrium between the protein subunits and spindle microtubules is unlikely to explain fully the behaviour of the spindle. Perhaps improvements in isolation technique will produce spindles that perform more like those in intact cells. Since exogenous enzymes, cofactors and inhibitors can gain access to the isolated spindles, it should be possible to analyse their role in spindle formation and disassembly. The spindle, however, is itself likely to contain a complex mixture of enzymes and other constituents, so that the analysis will not be straightforward.

Gibbons and others have found how useful sperm tail preparations made accessible to exogenous agents by detergents can be in analysing flagellar movement, and similar analyses should be possible with isolated spindles. It would be of great interest to know whether spindle assembly or disassembly depends on reversible polymerisation of tubulin and whether the presence of dynein, actin or some other contractile protein is required for chromosome movement. Apparently low calcium is necessary for assembly, as well as phosphorylation of GDP tightly bound to tubulin. Jacobs at King's College London, has recently been studying a transphosphorylase associated with tubulin, but separable from it, which seems to be involved in the assembly process. Among the many possible systems involved in the control of spindle assembly and action are the concentration of calcium and level of transphosphorylase activity. 